\title{
CARRIER PHASE INTEGER AMBIGUITY RESOLUTION WITH INEQUALITY CONSTRAINTS FOR GPS AND GALILEO
}

\author{
Patrick Henkel and Chen Zhu \\ Institute for Communications and Navigation \\ Technische Universität München, Munich, Germany
}

\begin{abstract}
Galileo and GPS receivers can measure the carrier phases with millimeter accuracy. However, the carrier phases are periodic which requires the resolution of an integer ambiguity for each satellite.

This paper provides two new constrained integer leastsquares estimators that include some a priori knowledge on the relative receiver position in spherical coordinates: a maximum a posteriori probability estimator and an inequality constrained integer ambiguity resolution. The latter one uses a polynomial barrier function. Both estimators enable a significant reduction of the integer search space, a substantial improvement in the float ambiguity estimates, and thereby, a higher probability of correct integer ambiguity resolution.
\end{abstract}

Index Terms - Carrier phase based positioning, integer ambiguity resolution, inequality constraints, barrier function, GPS and Galileo.

\section{INTRODUCTION}

GPS and Galileo receivers use both the code and carrier phases for positioning. The carrier phase can be tracked with millimeter accuracy but it is periodic, and requires the resolution of an integer ambiguity for each satellite. The differential positioning of a receiver with respect to a reference station enables a resolution of these ambiguities as atmospheric errors and satellite biases can be canceled by double differences.

Fig. 1 shows the wavefronts from three satellites, which intersect in the true receiver position. The pure code solution provides a rough estimate of the receiver position, which leads to a certain search space volume (shown as circle). Obviously, the introduction of some a priori knowledge on the length and orientation of the baseline further constrains the search space.

The Least-squares Ambiguity Decorrelation Adjustment (LAMBDA) method was developed by Teunissen in [1] to solve the unconstrained integer least-squares estimation. He introduced an integer ambiguity transformation based on an alternating sequence of permutations and integer decorrelations to obtain a sphere-like and largely decorrelated search space. In [2], Teunissen provided an integer least-squares estimator with a hard constraint on the baseline length. He

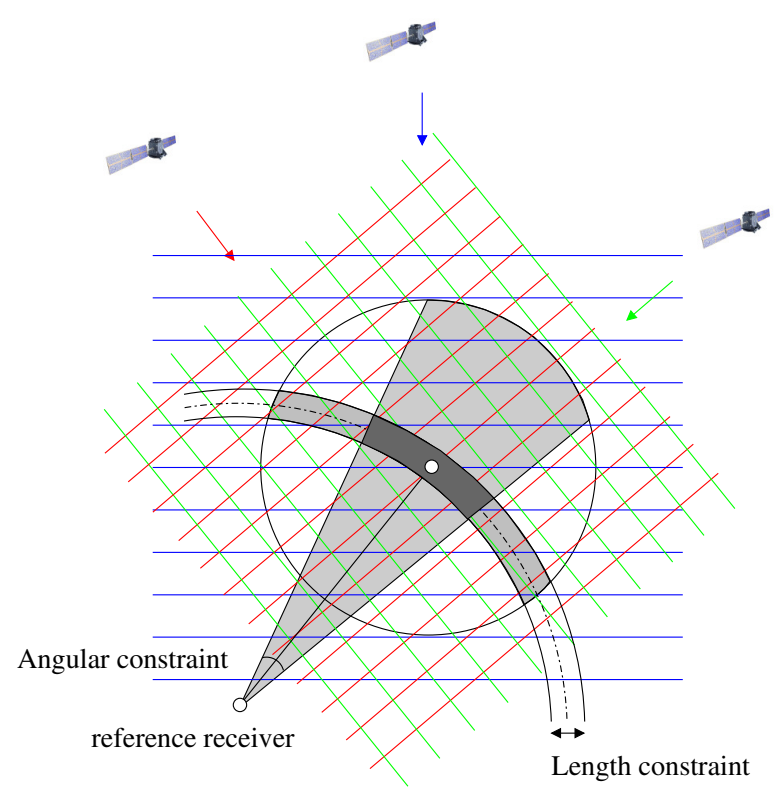

Fig. 1. Integer ambiguity grid: The search space volume of the float solution is reduced by spatial constraints on the receiver position with respect to a reference station.

extended his constraint to a "soft" Gaussian one in [3]. However, he did not include any constraints on the baseline orientation. This is a non-trivial extension as the angles describing the baseline direction enter the cost function in a highly nonlinear form. However, soft constraints on the direction are extremely helpful to reduce the size of the search space and, thereby, to improve the reliability of ambiguity resolution.

This paper is organized as follows: Section II includes our system model with a spherical parameterization of the baseline coordinates. A maximum a posteriori probability estimator with Gaussian a priori knowledge of the baseline length and orientation is introduced in Section III. It enables a substantial reduction of the probability of wrong fixing compared to unconstrained ambiguity resolution. Section IV includes a second soft constrained integer ambiguity resolution, which includes some inequality constraints by a polynomial barrier function. Finally, Section V summarizes the paper. 


\section{SYSTEM MODEL}

The double difference carrier phase measurements on frequency $f_{m}$ of satellite $k \in\{1, \ldots, K\}$ are modeled as

$$
\begin{aligned}
\nabla \Delta \phi_{m}^{k}= & \nabla \Delta r^{k}+\lambda_{m} \nabla \Delta N_{m}^{k} \\
& -q_{1 m}^{2} \nabla \Delta I^{k}+\Delta T^{k}+\nabla \Delta \eta_{\phi_{m}}^{k},
\end{aligned}
$$

with the double difference range $\nabla \Delta r^{k}$, the double difference integer ambiguity $\nabla \Delta N_{m}^{k}$, the double difference ionospheric and tropospheric delays $\nabla \Delta I^{k}$ and $\nabla \Delta T^{k}$, the ratio of frequencies $q_{1 m}=f_{1} / f_{m}$, and the double difference measurement noise $\nabla \Delta \eta_{\phi_{m}}^{k}$. Obviously, the double difference atmospheric delays can be neglected for short baselines as considered in this paper. A similar model is used for the double difference code measurements, i.e.

$$
\nabla \Delta \rho_{m}^{k}=\nabla \Delta r^{k}+q_{1 m}^{2} \nabla \Delta I^{k}+\nabla \Delta T^{k}+\nabla \Delta \eta_{\rho_{m}}^{k}
$$

Two multi-frequency linear combinations are applied to the double difference carrier phase measurements $\lambda_{m} \nabla \Delta \phi_{m}$ and code measurements $\nabla \Delta \rho_{m}$ : a code carrier linear combination and a code-only combination, i.e.

$$
\boldsymbol{\Psi}=\left[\begin{array}{c}
\sum_{m=1}^{M}\left(\alpha_{m} \lambda_{m} \nabla \Delta \boldsymbol{\phi}_{m}+\beta_{m} \nabla \Delta \boldsymbol{\rho}_{m}\right) \\
\sum_{m=1}^{M}\left(\beta_{m}^{\prime} \nabla \Delta \boldsymbol{\rho}_{m}\right)
\end{array}\right],
$$

where $\alpha_{m}$ denote the weighting coefficients of the phase measurements, and $\beta_{m}$ and $\beta_{m}^{\prime}$ represent the code coefficients on frequency $m \in\{1, \ldots, M\}$. These coefficients were optimized by Henkel et al. in [5]-[7] such that the linear combinations preserve the non-dispersive range (i.e. $\left.\sum_{m=1}^{M}\left(\alpha_{m}+\beta_{m}\right)=1\right)$, eliminate the ionospheric delay (i.e. $\left.\sum_{m=1}^{M}\left(\alpha_{m}-\beta_{m}\right) q_{1 m}^{2}=0\right)$, keep the integer nature of ambiguities (i.e. $\sum_{m=1}^{M} \alpha_{m} \lambda_{m} N_{m}=\lambda N$ ), and maximize the ambiguity discrimination defined as the ratio between the combination wavelength and combination noise standard deviation.

The combined measurements $\boldsymbol{\Psi}$ are modeled as

$$
\boldsymbol{\Psi}=\boldsymbol{H} \boldsymbol{\xi}+\boldsymbol{A} \boldsymbol{N}+\boldsymbol{b}+\boldsymbol{\varepsilon}, \quad \boldsymbol{\xi} \in \mathbb{R}^{3 \times 1}, \boldsymbol{N} \in \mathbb{Z}^{K \times 1},
$$

where $\boldsymbol{H}$ describes the differential geometry given by

$$
\boldsymbol{H}=\left[\begin{array}{c}
\left(\boldsymbol{e}^{1}\right)^{T}-\left(\boldsymbol{e}^{K}\right)^{T} \\
\vdots \\
\left(\boldsymbol{e}^{K-1}\right)^{T}-\left(\boldsymbol{e}^{K}\right)^{T}
\end{array}\right]
$$

with the unit vector $\boldsymbol{e}^{k}$ pointing from the $k$-th satellite to the receiver, and $\boldsymbol{\xi}$ being the baseline between both receivers. It can be represented in spherical coordinates by two angles $\nu_{1}$ and $\nu_{2}$ and the length $l$, i.e.

$$
\boldsymbol{\xi}=\boldsymbol{r}\left(\nu_{1}, \nu_{2}\right) \cdot l,
$$

with

$$
\boldsymbol{r}\left(\nu_{1}, \nu_{2}\right)=\left[\begin{array}{c}
\cos \left(\nu_{1}\right) \cos \left(\nu_{2}\right) \\
\cos \left(\nu_{1}\right) \sin \left(\nu_{2}\right) \\
\sin \left(\nu_{1}\right)
\end{array}\right]
$$

The second term in (4) represents the combined integer ambiguities $N$ with the combination wavelength included in prefactor matrix $A$ :

$$
\boldsymbol{A}=\left[\begin{array}{c}
\lambda \cdot \boldsymbol{I} \\
\mathbf{0}
\end{array}\right]
$$

The unknown biases $\boldsymbol{b}$ describe multipath with long decorrelation time, and are introduced to test the robustness of the constrained ambiguity resolution. The measurement noise $\varepsilon \sim \mathcal{N}(\mathbf{0}, \mathbf{\Sigma})$ is assumed to be white Gaussian noise.

\section{MAXIMUM A POSTERIORI PROBABILITY ESTIMATION OF INTEGER AMBIGUITIES}

The constrained ambiguity resolution can be considered as a maximum likelihood (ML) estimation or as a maximum a posteriori probability (MAP) estimation. The latter one maximizes the a posteriori probability of the estimates of $\nu_{1}, \nu_{2}$ and $l$ for a given set $\boldsymbol{\Psi}$. This maximization is rewritten with the rule of Bayes and the assumption of statistically independent $\nu_{1}, \nu_{2}$ and $l$ as

$$
\begin{array}{rl}
\max _{\nu_{1}, \nu_{2}, l} & p\left(\nu_{1}, \nu_{2}, l \mid \boldsymbol{\Psi}\right) \\
& =\max _{\nu_{1}, \nu_{2}, l} p\left(\boldsymbol{\Psi} \mid \nu_{1}, \nu_{2}, l\right) \cdot \frac{p\left(\nu_{1}\right) p\left(\nu_{2}\right) p(l)}{p(\boldsymbol{\Psi})}
\end{array}
$$

where the conditional probability density is obtained from

$$
p\left(\boldsymbol{\Psi} \mid \nu_{1}, \nu_{2}, l\right)=\frac{1}{\sqrt{(2 \pi)^{2}|\boldsymbol{\Sigma}|}} e^{-\frac{1}{2}\left\|\boldsymbol{\Psi}-\boldsymbol{H} \boldsymbol{r}\left(\nu_{1}, \nu_{2}\right) l-\boldsymbol{A} \boldsymbol{N}\right\|_{\boldsymbol{\Sigma}}^{2}-1}
$$

The a priori knowledge is assumed to be Gaussian distributed with known means and variances, i.e.

$$
\begin{aligned}
p\left(\nu_{x}\right) & =\frac{1}{\sqrt{2 \pi \sigma_{\bar{\nu}_{x}}^{2}}} e^{-\frac{\left(\nu_{x}-\bar{\nu}_{x}\right)^{2}}{2 \sigma_{\nu_{x}}^{2}}}, \quad x \in\{1,2\} \\
p(l) & =\frac{1}{\sqrt{2 \pi \sigma_{\bar{l}}^{2}}} e^{-\frac{(l-\bar{l})^{2}}{2 \sigma_{l}^{2}}},
\end{aligned}
$$

and the probability density function of the measurements is obtained from (10) by

$$
p(\boldsymbol{\Psi})=\iiint p\left(\boldsymbol{\Psi} \mid \nu_{1}, \nu_{2}, l\right) p\left(\nu_{1}\right) p\left(\nu_{2}\right) p(l) d \nu_{1} d \nu_{2} d l
$$

The maximization of (9) can be simplified by taking the logarithm and omitting the pre-factor that does not depend on $\nu_{1}$, 
$\nu_{2}$ and $l$, i.e.

$$
\begin{aligned}
& \min _{\nu_{1}, \nu_{2}, l, \boldsymbol{N}} J\left(\nu_{1}, \nu_{2}, l, \boldsymbol{N}\right) \\
& =\min _{\nu_{1}, \nu_{2}, l, \boldsymbol{N}}\left(\left\|\boldsymbol{\Psi}-\boldsymbol{H} \boldsymbol{r}\left(\nu_{1}, \nu_{2}\right) l-\boldsymbol{A} \boldsymbol{N}\right\|_{\boldsymbol{\Sigma}^{-1}}^{2}\right. \\
& \left.\quad+\frac{(l-\bar{l})^{2}}{\sigma_{\bar{l}}^{2}}+\frac{\left(\nu_{1}-\bar{\nu}_{1}\right)^{2}}{\sigma_{\bar{\nu}_{1}}^{2}}+\frac{\left(\nu_{2}-\bar{\nu}_{2}\right)^{2}}{\sigma_{\overline{\nu_{2}}}^{2}}\right) .
\end{aligned}
$$

This optimization is split into a tree search of $\boldsymbol{N}$ and an iterative computation of $\nu_{1}, \nu_{2}$ and $l$ for each candidate $N$. The integer search aims on finding all integer vectors $N$ that fulfill

$$
\|\hat{\boldsymbol{N}}-\boldsymbol{N}\|_{\boldsymbol{\Sigma}_{\hat{\mathbf{N}}}^{-1}}^{2} \leq \chi^{2},
$$

where $\hat{\boldsymbol{N}}=\left(\overline{\boldsymbol{A}}^{T} \boldsymbol{\Sigma}^{-1} \overline{\boldsymbol{A}}\right)^{-1} \overline{\boldsymbol{A}}^{T} \boldsymbol{\Sigma}^{-1} \boldsymbol{\Sigma}^{-1} \boldsymbol{\Psi}$ denotes the unconstrained float solution with the projected coefficient matrix $\overline{\boldsymbol{A}}=\boldsymbol{P}_{H}^{\perp} \boldsymbol{A}=\left(\mathbf{1}-\boldsymbol{H}\left(\boldsymbol{H}^{T} \boldsymbol{\Sigma}^{-1} \boldsymbol{H}\right)^{-1} \boldsymbol{H}^{T} \boldsymbol{\Sigma}^{-1}\right) \boldsymbol{A}$, and $\chi^{2}$ is the search space volume. Teunissen introduced an ambiguity transformation in [1] to decorrelate the search space and to optimize the order of ambiguities. The efficiency of the search can be further improved if the baseline constraints are also included in the search tree as described by Jurkowski et al. in [4].

The baseline parameters are determined iteratively with the Newton method. In the $n$-th step, the baseline parameters are given by

$$
\left[\begin{array}{c}
\hat{\nu}_{1}^{n+1} \\
\hat{\nu}_{2}^{n+1} \\
\hat{l}^{n+1}
\end{array}\right]=\left[\begin{array}{c}
\hat{\nu}_{1}^{n} \\
\hat{\nu}_{2}^{n} \\
\hat{l}^{n}
\end{array}\right]-\boldsymbol{S}^{-1}\left[\begin{array}{c}
\frac{\partial J}{\partial \nu_{1}} \\
\frac{\partial J}{\partial \nu_{2}} \\
\frac{\partial J}{\partial l}
\end{array}\right],
$$

where $\hat{\nu}_{x}, x \in\{1,2\}$, and $\hat{l}$ include the estimates of all epochs, and the Hesse matrix is given by

$$
\boldsymbol{S}=\left[\begin{array}{ccc}
\frac{\partial^{2} J}{\partial \nu_{1}^{2}} & \frac{\partial^{2} J}{\partial \nu_{1} \partial \nu_{2}} & \frac{\partial^{2} J}{\partial \nu_{1} \partial l} \\
\frac{\partial^{2} J}{\partial \nu_{1} \partial \nu_{2}} & \frac{\partial^{2} J}{\partial \nu_{2}^{2}} & \frac{\partial^{2} J}{\partial \nu_{2} \partial l} \\
\frac{\partial^{2} J}{\partial \nu_{1} \partial l} & \frac{\partial^{2} J}{\partial \nu_{2} \partial l} & \frac{\partial^{2} J}{\partial l^{2}}
\end{array}\right] .
$$

As the cost function of (13) is not convex, the Newton method is initialized with $\nu_{1}, \nu_{2}$ and $l$ from a systematic grid.

Fig. 2 shows the benefit of a tight and soft length constraint for integer least-squares estimation (ILS) with Galileo. Double difference measurements on E1 and E5 were simulated for a baseline length of $30 \mathrm{~m}$, a geometry with 8 visible satellites, 4 measurement epochs, and a standard deviation of $1 \mathrm{~mm}$ for the phase noise. Phase-only measurements were considered to avoid code multipath, and a widelane combination with a wavelength of $78.1 \mathrm{~cm}$ was chosen to increase the success rate. Obviously, the tightly constrained (TC) ambiguity resolution reduces the probability of wrong unconstrained fixing by more than four orders of magnitude if the a priori length information is correct. However, it makes the fixing also sensitive w.r.t. erroneous a priori information, i.e. it degrades the unconstrained performance if the error in the a priori information exceeds $50 \mathrm{~cm}$. The soft constrained (SC) fixing takes the uncertainty in the length information into account and, thereby, improves the unconstrained fixing for any quality of the a priori information. Note that the TC ambiguity resolution is obtained from the SC one by setting $\sigma_{\hat{l}} \rightarrow \infty$. It can also be obtained from Lagrange optimization with an equality constraint.

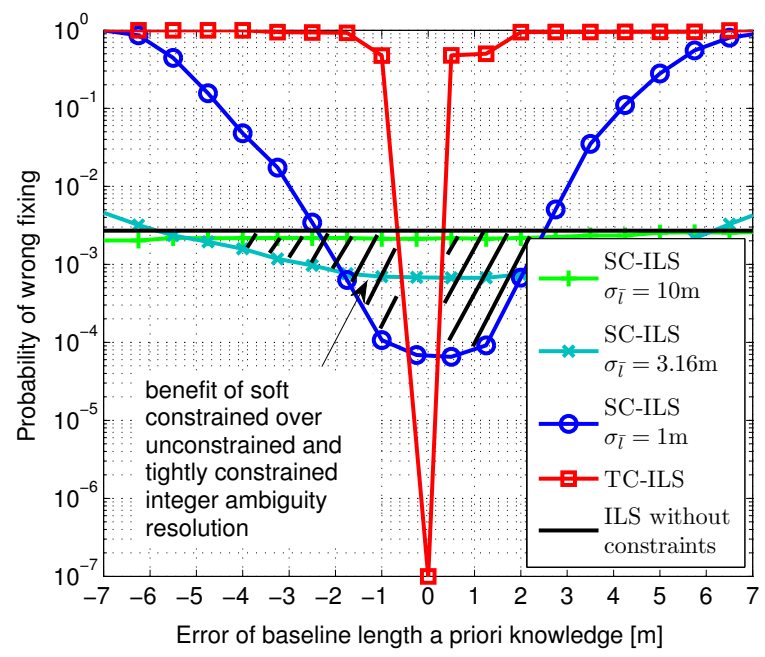

Fig. 2. Comparison of unconstrained, soft constrained and tightly constrained ambiguity resolution for erroneous baseline length a priori information: The tightly constrained ambiguity resolution outperforms the unconstrained and soft constrained fixing for perfect a priori knowledge but is extremely sensitive w.r.t. erroneous a priori information. The soft constrained ambiguity fixing benefits from the a priori information even if it is biased.

\section{ROBUST INTEGER AMBIGUITY RESOLUTION WITH INEQUALITY CONSTRAINTS}

The maximum a posteriori probability estimator of the previous section enabled a substantial improvement in ambiguity fixing as it benefits from an a priori known Gaussian probability distribution of the baseline parameters. However, if this information is erroneous or unavailable, inequality constraints can be very beneficial and lead to the minimization

$$
\begin{gathered}
\min _{\nu_{1}, \nu_{2}, l, \boldsymbol{N} \in \mathbb{Z}^{K}}\left\|\boldsymbol{\Psi}-\boldsymbol{H} \boldsymbol{r}\left(\nu_{1}, \nu_{2}\right) l-\boldsymbol{A} \boldsymbol{N}\right\|_{\boldsymbol{\Sigma}^{-1}}^{2} \\
\text { s.t. } \quad \nu_{1, \min } \leq \nu_{1} \leq \nu_{1, \max }, \\
\nu_{2, \min } \leq \nu_{2} \leq \nu_{2, \max }, \\
l_{\min } \leq l \leq l_{\max },
\end{gathered}
$$

which can also be regarded as uniform distributions. The inequality constrained ambiguity resolution can be approxi- 
mated by an unconstrained optimization problem, i.e.

$$
\begin{gathered}
\min _{\nu_{1}, \nu_{2}, l, \boldsymbol{N} \in \mathbb{Z}^{K}}\left(\left\|\boldsymbol{\Psi}-\boldsymbol{H} \boldsymbol{r}\left(\nu_{1}, \nu_{2}\right) l-\boldsymbol{A} \boldsymbol{N}\right\|_{\boldsymbol{\Sigma}^{-1}}^{2}\right. \\
\left.+\sum_{i=1}^{2} f\left(\nu_{i}, \nu_{i, \min }, \nu_{i, \max }\right)+f\left(l, l_{\min }, l_{\max }\right)\right)
\end{gathered}
$$

with the barrier function

$$
f\left(x, x_{\min }, x_{\max }\right)= \begin{cases}t \cdot\left(x_{\min }-x\right)^{h} & x<x_{\min }, \\ 0 & x_{\min } \leq x \leq x_{\max } \\ t \cdot\left(x-x_{\max }\right)^{h} & x>x_{\max }\end{cases}
$$

with penalty factor $t$ and $h \geq 3$. This barrier function is second order continuously differentiable so that the minimization of (18) can be again solved iteratively with the Newton method. For $t \rightarrow \infty$, (18) becomes equivalent to (17).

Fig. 3 shows the benefit of the inequality constraints for float ambiguity estimation as a function of the tightness of the bounds. For example, restricting the elevation angle to an interval of $\delta \nu_{1}=10^{\circ}$, reduces the uncertainty in the float ambiguity estimates by a factor two compared to $\delta \nu_{1}=50^{\circ}$.

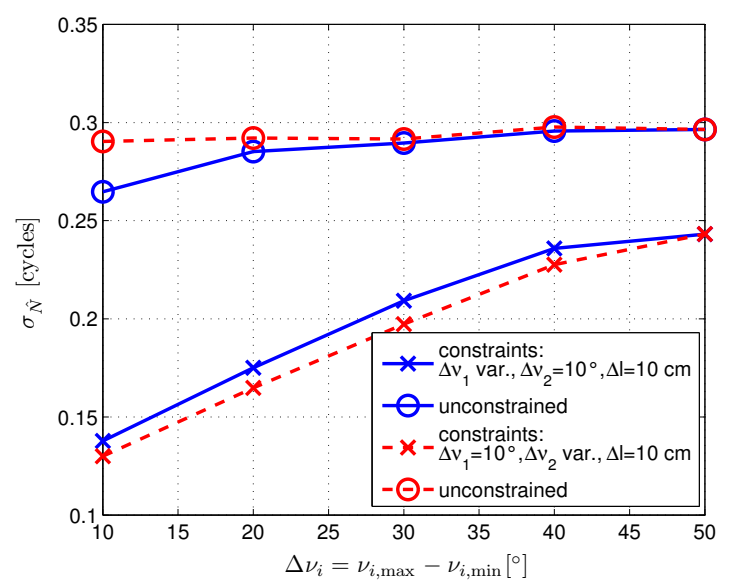

Fig. 3. Benefit of inequality constrained integer ambiguity resolution over unconstrained ambiguity resolution

The float ambiguity estimates can be fixed either by a search or rounding. The latter one is in general performed sequentially (e.g. [1]) to take the correlation between the float ambiguities into account, i.e. the float conditional estimate of the second ambiguity is given by

$$
\hat{N}_{2 \mid 1}=\hat{N}_{2}-\gamma \cdot\left(\hat{N}_{1}-\left[\hat{N}_{1}\right]\right),
$$

where $[\cdot]$ denotes the rounding operator and $\gamma$ is obtained by minimizing the variance of $\hat{N}_{2 \mid 1}$, i.e.

$$
\gamma_{\mathrm{opt}}=\frac{\sigma_{\hat{N}_{1} \hat{N}_{2}}-\sigma_{\left[\hat{N}_{1}\right] \hat{N}_{2}}}{\sigma_{\hat{N}_{1}}^{2}-2 \sigma_{\hat{N}_{1}\left[\hat{N}_{1}\right]}+\sigma_{\left[\hat{N}_{1}\right]}^{2}},
$$

where the covariance between $\left[\hat{N}_{1}\right]$ and $\hat{N}_{2}$ is given by

$$
\sigma_{\left[\hat{N}_{1}\right] \hat{N}_{2}}=-\frac{\sigma_{\hat{N}_{1} \hat{N}_{2}}}{\sqrt{2 \pi \sigma_{\hat{N}_{1}}^{2}}} \sum_{k=-\infty}^{+\infty} k\left(e^{-\frac{(k+0.5)^{2}}{2 \sigma_{\hat{N}_{1}}^{2}}}-e^{-\frac{(k-0.5)^{2}}{2 \sigma_{\hat{N}_{1}}^{2}}}\right)
$$

which can be well approximated by a finite sum.

\section{CONCLUSION}

In this paper, the reliability of carrier phase based positioning with integer ambiguity resolution was improved by including some soft constraints on the receiver position. This a priori knowledge is typically given either as Gaussian distributions or inequality constraints on the spherical position coordinates. For the first case, a maximum a posteriori probability estimator was derived based on the iterative Newton method. For the second case, the inequality constraints were included in the cost function by a polynomial barrier function.

The maximum a posteriori probability estimator enables a probability of wrong fixing that is several orders of magnitude lower than for unconstrained ambiguity resolution even if the a priori information is slightly biased. The inequality constrained fixing benefits from a substantial reduction of the integer search space and an improved accuracy of the float solution, which turns into a lower probability of wrong fixing.

\section{REFERENCES}

[1] P. Teunissen, "The least-squares ambiguity decorrelation adjustment: a method for fast GPS integer ambiguity resolution”, J. of Geodesy, vol. 70, pp. 65-82, 1995.

[2] P. Teunissen, "The LAMBDA method for the GNSS compass", Art. Satellites, vol. 41, nr. 3, pp. 89-103, 2006.

[3] P. Teunissen, "Integer least-squares theory for the GNSS compass", J. of Geodesy, vol. 84, pp. 433-447, 2010.

[4] P. Jurkowski, P. Henkel, G. Gao and C. Günther, "Integer Ambiguity Resolution with Tight and Soft Baseline Constraints for Freight Stabilization at Helicopters and Cranes", Proc. of ION Intern. Techn. Meet., San Diego (USA), Jan. 2011.

[5] P. Henkel and C. Günther, "Joint L-/C-Band Code and Carrier Phase Linear Combinations for Galileo", Int. J. of Nav. and Obs., pp. 1-8, 2008.

[6] P. Henkel, "Bootstrapping with Multi-Frequency Mixed Code Carrier Linear Combinations and Partial Integer Decorrelation in the Presence of Biases", Proc. of Int. Assoc. of Geod. Scient. Ass., Buenos Aires, 2009.

[7] P. Henkel and C. Günther, "Reliable Carrier Phase Positioning with Multi-Frequency Code Carrier Linear Combinations", Proc. of 23rd ION Intern. Techn. Meet. (IONGNSS), Portland, USA, pp. 185-195, 2010. 